\title{
The Michel Benoist and Robert Mulholland yearly European Spine Journal Review: a survey of the "medical" articles in the European Spine Journal, 2010
}

\author{
Michel Benoist
}

Received: 22 December 2010/Published online: 8 January 2011

(C) Springer-Verlag 2011

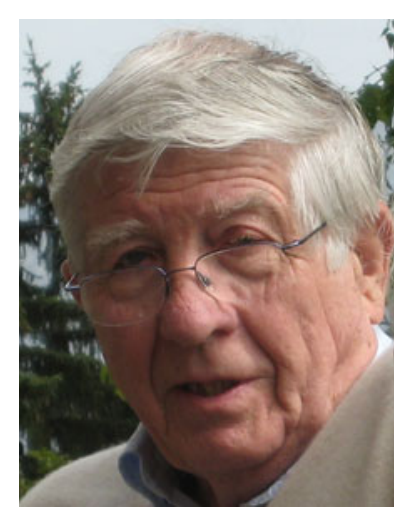

\section{Introduction}

From the wealth of information provided in the European Spine Journal in 2010, I have tried to extract the most "medically oriented" papers. As usual, there are fewer "medical" than "surgical" articles. During my selection, I realized that the distinction between surgical and nonsurgical papers was an arbitrary one. Articles dealing with pure surgical techniques are obviously of little interest to the medical readers, yet the numerous non-surgical spinal specialists must be aware of the development of new surgical procedures. Confirmation with the passage of time of the validity of less recent surgical therapies, with their risk of complications, is also essential to a proper counseling of the patient. It is equally important for the spinal surgeon, who is frequently the first-line consultant, to have a clear

\section{Benoist ( $\square)$}

Département de Rhumatologie, Service de Chirurgie

Orthopédique, Hôpital Beaujon, 100 Boulevard Général Leclerc, 92118 Clichy, France

e-mail: deuxmice@aol.com estimation of the therapeutic value of the various conservative treatment modalities. In addition, care of patients with spinal diseases often calls for a multi-disciplinary approach. This emphasizes the need for all of us to be informed of the progress of basic research, and of the everevolving technologies, such as, for example imaging and electrophysiological techniques. I therefore invite the reader to have a look at both reviews, medical and surgical. He may well find that the same papers have occasionally been reviewed by both Bob Mulholland and myself.

\section{Basics}

Two interesting research articles can be found in the October issue. The experimental paper by Hirose et al. [9] deals with the pathomechanisms of pain following mechanical compression of a peripheral nerve. In this model, the sciatic nerve of rats was pinched with a forceps in 20 rats; in 20 other rats, the sciatic nerve was exposed but not pinched and used as a control group. Mechanical and thermal hyperalgesia were evaluated in the two groups. On days 4 and 7, dorsal root ganglions were resected and immuno-histochemical staining performed for detection of neuropeptide markers of pain. Mechanical and thermal hyperalgesia were observed in the pinched group and not in the sham group. Immunoreactivity was upregulated for pain marker expression in the nerve injury group, suggesting that this simple model could be used as a model of pain for short-term studies.

The article by Min et al. [17] provides a useful insight into proliferation and osteoblastic differentiation of bone marrow from stem cells (BMSCs). The aim of this study published in the October issue was to compare BMSCs obtained from the vertebral body (VB) with those harvested 
from the iliac crest (IC). Using appropriate and sophisticated biochemical and molecular biology techniques, proliferation and osteoblastic differentiation of each of the BMSCs are characterized and compared. The results of the study indicate that a sufficient volume of bone marrow aspirate can be obtained from the vertebral body. Most importantly, VB and IC bone marrow have similar characteristic properties with regard to the number of mononuclear cells capable of differentiating into osteoblasts, of the concentration of BMSCs and osteogenic expression. The authors conclude that VB bone marrow could provide adequate stem cells for graft augmentation in spinal fusion surgery, thus diminishing the need for bone marrow harvested in the iliac crest.

An interesting experimental paper conducted by Koshi et al. [15] published in the April issue explores the mechanisms of pain relief following lumbar postero-lateral fusion (PLF). The experimental procedure comprises disc puncture (creating degeneration) and application of fluorogold (to detect DRG neurons innervating the intervertebral disc) in 20 rats. Ten of these (group A) had a sham PLF and ten (group B) had a PLF. A control group of 20 other rats comprised 10 with no puncture with PLF (group C) and 10 with no puncture and sham PLF (group D). Four weeks after surgery, bilateral L1-L5 dorsal root ganglia were stained with growth-associated protein 43 (GAP 43), a marker of axonal growth and calcitonin gene-related peptide (CGRP), a marker of pain. Bone union analysis of the results disclosed that postero-lateral fusion is strongly related to a down regulation of GAP 43 and of CGRP. Suppression of the increase of inflammatory neuropeptides and of axonal growth may explain the mechanism of pain relief following PLF. The validity of application of these results to humans is questionable and is thoroughly discussed by the authors.

\section{Functional restoration programs for chronic low-back pain}

The article by Poulain et al. [23] in the July issue examines the long-term effectiveness of a functional restoration program (FRP) in a group of 170 motivated patients with an invalidating chronic low-back pain (LBP) lasting over 3 months, and with more than 1 month of absenteeism caused by LBP. The primary end point is return to work. Predictive factors of long-term return to work are also assessed. The paper describes in detail the program of restoration, which is not always the case in other articles of this category. A thorough and appropriate evaluation of the medical, professional, social, and psychological conditions is assessed at base line by medical evaluation and selfquestionnaires. The same evaluation is performed at 1,6 , and 12 months and at the final follow-up, which comprised only 105 patients, due to 58 patients having been lost to follow-up. The results are particularly interesting considering the profile of the patients: $55 \%$ returned to work after a mean follow-up of 3.5 years. Quality of life, functional disability, as well as all the psychological variables were improved. In the discussion, these results are compared with similar studies of the literature, providing a good update of these costly programs. The authors very wisely indicate that the results must be tempered with the high number of non-responders at the final follow-up.

A similar study performed in UK by van Hooff et al. [28] and reported in the September issue has evaluated the midterm effects of an intensive cognitive behavioral program in patients with chronic LBP. One hundred and seven patients participated in the program. Main outcome parameters were daily functioning (Roland and Morris Disability Questionnaire and Oswestry Disability Questionnaire), self-efficacy questionnaire, and quality of life (short form 36PCS). All questionnaires were assessed at base line on the last day of the residential program, and at 1 and 12 months. Results are evaluated with an excellent methodology and are compared with other multidisciplinary programs and with the results of fusion surgery. Overall, more than half of the cohort improved in daily functioning, and $80 \%$ improved very significantly in their quality of life. These beneficial results persisted at the 1-year follow-up and were greater than the reference MCID for all measurements. Results of this study are also comparable with those of fusion for chronic low-back pain patients, even for those with a long duration of pain: the mean duration of LBP in this study was 12 years. As suggested by the authors, future research should be directed to identify which patients should be selected for such programs.

\section{Cervical myelopathy}

In the May issue, Scardino et al. [26] have evaluated the possible benefits of surgery in patients with severe advanced-stage cervical spondylotic myelopathy. They retrospectively analyzed a subgroup of patients: seriously ill, bedridden, or in a wheelchair with a Nurick score equal to 5. All spinal cords presented high-intensity signal on T2 weighted images. This subgroup comprised 9 patients extracted from an overall operated group of 55 patients. Their mean age was around 70 years and the mean postoperative follow-up was $53.44 \pm 35.09$ months. Interestingly, six out of the nine patients achieved a substantial functional improvement. More specifically, they regained the ability to walk. The good clinical improvement was correlated with at least a gain of one degree on the Nurick 
scale post-operatively. The more sensitive JOAm score was 6.4 preoperatively and 9.88 after surgery. There is a good review of the literature concerning this type of patient. Spinal cord atrophy on MRI and prolonged length of disease are predictors of poor outcomes.

In the same issue of the Journal, Jameson et al. [11] published an interesting and useful report with a literature review of cervical myelopathy in patients with athetoid and dystonic cerebral palsy. The authors have operated seven such patients, performing cord decompression and fusion. A detailed analysis of the seven cases is provided. The initial symptoms comprising spasticity, limb weakness, paresthesia, and sphincter dysfunction develop progressively in the context of abnormal movements and dysarthria, which explains the difficulty of detecting the onset of myelopathy. In such young patients, generally in the fourth decade, the initial neurologic symptoms and signs of cord disturbance must be carefully and regularly checked in order to avoid a long delay of diagnosis. Decrease of abnormal movements is an alarming sign, which must not be interpreted as an improvement of the dystonic condition. In the present series, cord compression consisted of two discal herniations and five stenoses. Awkward posture and repeated abnormal movements of the cervical spine explain, at least in part, the prevalence of myelopathy in this pathology. All patients of this series improved functionally, post-operatively, with a mean gain of 1.5 points of the JOAm score. Difficulties and complications of this surgery are thoroughly discussed as well as the cases of the literature.

\section{Imaging}

Recently, an abnormal load transfer to the underlying end plate and vertebra has been compared to the high-spot loading of a subluxing hip. It was suggested that abnormal loading distribution could be the source of LBP [18]. It could therefore be speculated that Modic changes could be related to an excessive pressure on the disc. Results of the study by Jensen et al. [13] in the January issue are not in line with this interpretation. The aim of the present study was to investigate the predictive value of lifestyle factors and MRI findings in the development of new Modic changes. In a prospective study the authors followed a cohort of 344 individuals who had an MRI and clinical evaluations at 40 years and again at 44 years. Multiple variables were analyzed including the MRI changes and lifestyle factors (physical work, leisure activity, BMI, smoking). New Modic changes, mainly of type 1, were found. Statistical analysis showed that the new Modic changes were principally related to the status of the disc at base line (degenerative, bulge, herniation, and other changes of the endplate) rather than to the lifestyle, including heavy physical load.

\section{Infection}

Ohtori et al. [20] have examined the prevalence of pyogenic spondylitis in patients with Modic I- type changes, reported in the July issue. Seventy-one patients with lowback pain and radiculopathy, and Modic I abnormalities on MRI were followed up for 2 years. At the initial examination all 71 patients were in good health with no fever, and laboratory studies (white blood cell count and level of C-reactive protein) were normal. Clinical examination, $\mathrm{X}$-ray of the lumbar spine, and laboratory studies were performed every 3 months and an MRI every year. During the follow-up, the clinical symptoms worsened in four patients who were clinically suspected to have an infection. This was confirmed by characteristic X-ray and MRI changes, blood results, and open biopsy. Microbiological studies disclosed an infection by low-virulent bacteria. In the discussion, the authors provide references in the literature supporting the fact that low-virulence microorganisms such as staphylococcus epidermis, propionibacterium acnes may develop slowly without evident signs of infection. This possibility must be considered in patients with low-back pain and Modic I changes when discussing treatment. The authors allude to the interest in doubtful cases of FDG-PET scans, which have been shown to be able to differentiate degenerative from infectious changes in patients with abnormalities detected on MRI. The discussion also includes a summary of the pathophysiology of the Modic changes.

An excellent update of the value of F-fluoro-D-deoxyglucose PET and PET/CT in the diagnosis of spinal infections was published in the April issue of the Journal. Gemmel et al. [7] first review the different morphological spine imaging, including conventional radiography and $\mathrm{CT}$, used to perform guided biopsy for histopathology and microbiology. Gadolinium-enhanced MRI has a high diagnostic accuracy but has limitations, especially in postoperative infection, hampered by the presence of metallic implants. The diagnostic value of the various conventional routinely used radionuclide techniques such as bone scintigraphy, labeled leucocytes, and gallium scanning is discussed and compared with the advantages and disadvantages of FDG PET. Published studies evaluating FDG PET and PET/CT in patients with spinal infections are extensively reviewed as well as the physical principle of this newly emerging technique. At the present time FDG PET is very costly, and appears to be especially helpful in patients where MRI cannot be performed (pace-makers, cardiac valves) or is non-diagnostic. 


\section{Conjoined lumbo-sacral nerve roots}

Preoperative diagnosis of conjoined nerve roots (CNR) versus discal herniation is important in order to avoid peroperative neural injury and surgical failures, as well as to plan an appropriate surgical treatment. The paper by Lotan et al. [16] in the July issue is a good reminder of the clinical and imaging features of this condition, not always recognized preoperatively. The authors report a series of 16 patients where CNR was confirmed intra-operatively, while in the same period 260 patients were surgically treated for a discal herniation. The CNR cohort was matched 1:2 with 32 patients with discal herniations. Clinical symptoms and signs, neurologic deficit, imaging studies, and surgical outcome were compared. Clinically, $44 \%$ of the patients had a neurogenic claudication, and less neurologic deficit than the disc herniation patients. Preoperatively, the surgeon suspected the correct diagnosis on the imaging studies in $40 \%$ of the cases. The radiological diagnosis of CNR is challenging, with some pictures resembling a discal herniation. According to the authors, the differential radiological diagnosis is principally based on equal density of the nerve-root anomaly and the dural sac, on asymmetry of the subarachnoid space in the axial view and on the location of the root anomaly above the disc space. Coronal reformations provide a good outline of the CNR. In the present series, the surgical treatment aiming at decompressing the lateral recess by laminectomy and foraminotomy was followed by outcomes similar to discal herniation surgery.

\section{Low-back pain in adolescents}

In the April issue, Balagué et al. [2] examine the association between isoinertial trunk muscle performance, using a tri-axial trunk dynamometer and consequential non-trivial LBP (requiring medical attention and/or limiting activities) in 95 male adolescents aged 13-14. History of LBP, including the last episode and family history were assessed as well as the type and frequency of sports activity. Trunk performance capacity was measured as range of motion, isometric strength, and maximal trunk velocity. Physical characteristics, sports habits, and various clinical variables, and trunk performance are compared in subjects with or without consequential LBP. LBP status was reassessed at 2 years' follow-up in $89 \%$ of the subjects. Overall statistical analysis of the results indicates that regular sports activity was associated with the presence of LBP, but not with isoinertial trunk muscle performance. The article provides a good discussion with a review of the literature concerning the value of trunk performance evaluation in relation to LBP, as well as to other predictors of prevalence and recurrent rates of LBP in adolescents. As trunk muscle performance appears to have no relation to LBP, the authors also suggest that these specific aspects have no logical place in the rehabilitation program.

\section{Tumors}

In the January issue, an interesting case of Langerhans cell histiocytosis located in the lateral mass of the atlas is reported by Zhong et al. [29] in the context of a Grand Round. Percutaneous CT-guided needle biopsy established the diagnosis of the osteolytic lesion. Conservative treatment including Halo vest immobilization and radiotherapy obtained a reconstruction of the lateral mass with a good clinical result at 7 years' follow-up. Following the case report there is a comprehensive and clear comment by Jouve [14] providing an update of the diagnosis and treatment of the condition. The author stresses the importance of the biopsy, even in the isolated typical presentation of a vertebra plana, in order to eliminate other possibly malignant etiologies. The necessity of a complete work-up in the search for another bone or visceral involvement is also stressed. Concerning treatment, the commentator also recommends spinal immobilization, surgery being rarely required. $\mathrm{He}$ is reluctant to advise radiotherapy, owing to the risk of malignant transformation.

\section{Electrophysiological studies}

The relevance of the use of motor-evoked potentials (MEPs) in the diagnosis and quantitative assessment of myelopathy patients has already been demonstrated. The interest of the study by Nakamae et al. [19] in the May issue is that this examination was performed in a large series of patients. The authors' purpose was to confirm and clarify the usefulness of MEPs as a screening tool for managing therapeutic indications. In this series, 831 patients with clinical symptoms and signs of myelopathy underwent MEPs measurement using transcranial magnetic stimulation. The cause of myelopathy varied, not only including spondylotic cervical myelopathy and discal herniation, but also tumors, ossification of the posterior longitudinal ligament, and of the yellow ligament. In addition to measuring the MEPs latency, the authors evaluated the central motor conduction time (CMCT), calculated by subtracting the peripheral conduction time from the MEP latency.

In this study, 349 patients were operated for myelopathy, and 482 with a less severe clinical symptomatology followed a conservative treatment. CMCTs in the operative group were significantly more prolonged than in the 
conservative group. This article confirms in a large series of patients that MEPs are useful in assessing the existence and severity of a myelopathy and are an important parameter when discussing surgery.

I would like to draw the attention of the reader to a new electrophysiological procedure described in the February issue by Quante et al. [24]. The paper contains a good description of the method, which has been shown to be able to detect damage of the dorsal root. In this study, the aim of the authors was to investigate the diagnostic value of laserevoked potentials (LEP) in acute radicular pain and to see whether the procedure performed soon after the onset of the acute pain could provide predictive parameters of prognosis. Fifty-four patients with acute sciatica related to a discal herniation on MRI were recruited. The laser stimuli are applied to the painful dermatome and to the contralateral healthy dermatome. The LEP parameters are described in detail in this paper. They consist schematically of a side-to-side difference of latency and amplitude transformed into a score. Comparison of the LEP parameters with the clinical evolution and outcome at 3 months allow an estimation of the predictive value. More precisely, in this series the latency difference between LEP of the painful side and the contralateral normal side was a predictor of the prognosis. If future studies from different centers confirm the results obtained in this group of patients, laser-evoked potentials would be particularly helpful in evaluating the efficacy of different therapies. LEP parameters could also, in addition to clinical and radiological data, help in deciding a surgical treatment earlier than usual.

\section{Disc herniation surgery}

Long-term result studies of discal surgery are few and indicate different success rates. The excellent study by Silverplats et al. [27] in the September issue provides an interesting insight and contains new information, especially concerning the predictive clinical factors of outcome. One hundred and seventy-five patients were evaluated at baseline, $154(90 \%)$ at 2 years, and $140(81 \%)$ at long term, around 7 years. Preoperative questionnaires collected baseline data; the potential predictors used were gender, age, smoking, level of the herniation, analgesics, length of sick leave, duration of leg pain, base-line leg and back pain, ODI, and the Zung depression scale. Patients were assessed at 2 years and at long-term follow-up, using the Macnab score and the patient's satisfaction for treatment. Overall, both objective and subjective evaluations reported approximately $70 \%$ good and excellent results. The statistical analyses of the predictors of outcome showed that preoperative duration of sick leave and of leg pain were the most important predictors. I strongly recommend careful reading of this important article. The information provided in this study has evident therapeutic consequences at the individual and societal levels.

The effect of application of local anesthetic and/or corticosteroid in managing pain after discectomy is controversial and a matter of debate. Because of this uncertainty, Bahari et al. [1] have performed a prospective blinded randomized control trial to evaluate the efficacy of local triamcinolone acetonide, bupivacaine, or a combination of the two agents, applied topically over the nerve root before closure. This study appears in the July issue. A homogenous group of 100 patients undergoing primary single-level lumbar discectomy was recruited and divided into four sub-groups: corticoid only, bupivacaine only, corticoid and bupivacaine, and control. Twenty-five patients were randomized into each group. Pain score (VAS) was assessed pre-operatively and on days 1 and 8 weeks post-operatively. Twenty-four hours post-operative opiate requirement and the duration of the patient's stay were evaluated and compared between the four groups. A significant difference was disclosed in the pain score on day 1 in the mean opiate requirement, and duration of the hospital stay in the combination corticosteroid and bupivacaine. The main possible bias of this paper relates to the fact that only the patient was blinded to randomization.

\section{Whiplash}

It is now accepted that whiplash-associated disorders combine physical, social, and psychological parameters. The role of depression as a result of the accident is well known and has already been studied by the authors of the present article [4]. In an interesting follow-up study published in the June issue, Phillips et al. [22] further explore the factors involved in the onset and evaluation of the depressive symptoms. Their inception cohort populationbased study includes a large number of participants ( $n$ : 3,452 ) representing a sub-group of patients who participated at all follow-ups: 6 weeks, 3, 6, 9, and 12 months. Assessment of depression was based on the psychometric properties of the Center for Epidemiologic Studies depression scale. Of the 3,452 patients who completed the six questionnaires, $48.3 \%$ had no symptoms of depression during the entire 1-year evolution. An initial depressive symptomatology, immediately following the accident but disappearing during the follow-up, was observed in $25.9 \%$ of the patients. Persistent, or recurrent depression was disclosed in the remaining patients. Factors involved in the initial episode of depression and those involved in the course of the 1-year follow-up are studied and discussed 
separately. Overall, however, the authors state: "The most important characteristics in identifying those who are likely to experience depression were: higher post-crash pain, sustaining fracture bone, presence of pain-crash anxiety and prior mental health problems". This study is particularly important owing to therapeutic consequences.

\section{Lumbar supports}

Currently, lumbar supports are provided as treatment to patients with LBP and sciatica for prevention, either primary with the aim of preventing onset of LBP, or secondary with the aim of preventing recurrent episodes. A recent Cochrane Systematic Review [12] concluded that there is moderate evidence (level B) that lumbar supports, compared with no orthoses, do not prevent LBP and are no more effective than other types of interventions. However, investigations of the effects of lumbar supports in secondary prevention indicate that subgroups of workers may be at reduced risk for recurrent episodes if wearing a support. Evidently, compliance to the wearing of the support is a key parameter to a positive result. For this reason, the study by Roelofs et al. [21] published in the September issue is particularly informative. In a previous study [25] the authors had found in a group of home-care workers that the use of lumbar supports reduced the number of days with LBP by $45 \%$, the average pain intensity by $13 \%$, and improved the functional status by $14 \%$. Adherence to treatment was good, as $78 \%$ of the group had used the support at least one-third of the days they experienced LBP. In this follow-up study, the authors aim at finding out the determinants of the intention to use the support in a similar sub-group of home-care workers, in order to learn why people are adherent. In order to obtain this insight, the authors used the ASE model including attitude (positive or negative), social influences (peers, clients, colleagues, etc.) and self-efficacy. The strongest predictor for compliance was a positive attitude towards lumbar supports; social support and self-efficacy played a minor role. The paper provides a useful discussion and points out the limitations and the therapeutic consequences.

\section{Caudal epidurals}

Epidural steroid injections are commonly used in the management of low-back pain with radiculopathy. Most open studies disclose positive results, and recent systematic reviews [6] have demonstrated their effectiveness in discal herniation and radiculitis and to a lower degree of scientific evidence in spinal stenosis as well. However, a correct position of the needle in the epidural space is mandatory.
The importance of X-ray guidance to avoid incorrect placement and inefficacy of the injection has been recently reinforced by the study by Barham and Hilton [3] published in the September issue. The authors have prospectively studied a consecutive case series of 137 patients treated by caudal steroid injections for sciatica due to discal herniation or stenosis. The position of the needle first sited by the surgeon through the sacral hiatus in the epidural space was assessed radiologically by image intensifier and after aspiration by injection of a contrast dye. In 102 of the 137 patients, the needle was correctly sited by the surgeon, resulting in a miss rate of $26 \%$. In addition, failure of the cephalad flow of the dye to the level of the documented pathology was $6 \%$. There was no significant difference in the accuracy of the needle placement between consultant surgeons and adequately trained middle-grade surgeons. This study demonstrates once again that epidurals must be performed with $\mathrm{X}$-ray guidance and epidurogram.

\section{The piriformis syndrome}

The piriformis syndrome related to irritation of the sciatic nerve by the piriformis muscle is somewhat similar to the Loch Ness monster... and its existence is still controversial. In the December issue, Hopayian et al. [10] have courageously attempted to perform a systematic review of the literature concerning this ill-defined syndrome. They were able to extract 51 individual case reports and three aggregated data studies, consisting of case series aggregated for all patients. The methodological quality of the eligible studies was generally poor. The more common clinical features found were buttock pain, tenderness of the greater sciatic notch, and aggravation of the pain through sitting and with maneuvers that increase piriformis muscle tension. According to the authors, the concurrence of several of these clinical features may suggest the role of the piriformis in patients with atypical histories, negativity of lumbar MRI, and with electrophysiological studies indicating a clear implication of the nerve trunk. In several cases the diagnosis was confirmed by local anesthetic and steroid injections into the piriformis muscle, or by abnormal MRI neurography or abnormal MRI images suggesting structural abnormalities of the muscle. The authors provide a table summarizing these few cases with the appropriate references. The table also summarizes the various mechanisms of impingement of the sciatic nerve by the piriformis muscle. Relief after surgery is reported in a small number of studies and only three studies reported a prolonged postoperative relief. This paper is the first systematic review providing a complete state of the art and indications for future research. One can expect that in the future, with 
progress in imaging and in electrophysiological studies, the role of the piriformis will be more easily demonstrated. This is not the case at the present time.

\section{Injection therapy and denervation procedures for chronic low-back pain}

The paper by Henschke et al. [8] published in the September issue is a systematic review of the efficacy of injection therapy (facets, epidural space, discs) and of denervation procedures (facet joints, intervertebral disc) in chronic low-back pain. Participants were adult subjects with LBP of over 3 months' duration, including subjects with radiculopathy or any other non-specific degenerative pathology such as osteoarthritis. This latter group (osteoarthritis) is clinically ill defined but probably corresponds to pure low-back pain without radiculopathy, the distinction being clinically important. Among the exclusion criteria are subjects with vertebral spinal stenosis. The methodology is that of a Cochrane Review, evaluating the risk of bias and the quality of evidence of the RCTs. Using their selection criteria, the authors included 27 studies in this review. A large number of studies were systematically excluded. The major cause of exclusion was related to the duration of LBP which was either not reported or less than 3 months. Overall, the authors conclude to a low to very low-quality evidence to support the use of injection therapy and denervation procedures. Although it is recognized that pain mechanisms are different in acute, subacute and chronic pain, one wonders why so many studies, often of good quality, were excluded. This remark particularly concerns epidural steroid injections, as only three studies of low-quality evidence, according to the authors, are considered. The selection strategy used by the authors is in contrast with that of previous Cochrane Reviews and of recent systematic reviews [5, 6] reporting different conclusions. One can also question the combining in the selection criteria of individuals suffering from pure lowback pain and those with radiculopathy, as well as the exclusion of patients with stenosis.

\section{References}

1. Bahari S, El-Dahab M, Cleary M et al (2010) Efficacy of triamcinolone and bupivacaine for pain after lumbar discectomy. Eur Spine J 19:1099-1103

2. Balagué F, Bibbo E, Mélot C et al (2010) The association between isoinertial trunk muscle performance and low back pain in male adolescents. Eur Spine J 19:624-632

3. Barham G, Hilton A (2010) Caudal epidurals: the accuracy of blind needle placement and the value of a confirmatory epidurogram. Eur Spine J 19:1479-1483
4. Caroll LJ, Cassidy JD, Côté P (2006) Frequency, timing and course of depressive symptomatology in Whiplash. Spine 31:E551-E556

5. Chou R, Atlas SJ, Stanos SP et al (2009) Nonsurgical interventional therapies for low-back pain: a review of the evidence for an American Pain Society Clinical Practice Guideline. Spine 34:1078-1093

6. Conn A, Buenaventura RM, Datta S et al (2009) Systematic review of caudal epidural injections in the management of chronic low-back pain. Pain Physician 12:109-135

7. Gemmel F, Rijk PC, Collins JM et al (2010) Expanding role of 18F-fluoro-D-deoxyglucose PET and PET/CT in spinal infections. Eur Spine J 19:540-551

8. Henschke N, Kuijpers T, Rubinstein SM et al (2010) Injection therapy and denervation procedures for chronic low-back pain: a systematic review. Eur Spine J 19:1425-1449

9. Hirose K, Iwakura N, Orita S et al (2010) Evaluation of behavior and neuropeptide markers of pain in a simple sciatic nerve-pinch pain model in rats. Eur Spine J 19:1746-1752

10. Hopayian K, Song F, Riera R et al (2010) The clinical features of the piriformis syndrome: a systematic review. Eur Spine J 19:2095-2109

11. Jameson R, Rech C, Garreau de Loubresse C (2010) Cervical myelopathy in athetoid and dystonic cerebral palsy: retrospective study and literature review. Eur Spine J 19:706-712

12. Jellema P, Van Tulder MW, Van Poppel MNM et al (2001) Lumbar supports for prevention and treatment of low-back pain: a systematic review within the framework of the Cochrane Back Review Group. Spine 26:377-386

13. Jensen TS, Kjaer P, Korsholm L et al (2010) Predictors of new vertebral endplate (Modic) changes in the general population. Eur Spine J 19:129-135

14. Jouve JL (2010) Expert's comment concerning Grand Rounds case entitled "Langerhans cell histiocytosis of the atlas in an adult" (by Wo Quan Zhong et al.). Eur Spine J 19:23-24

15. Koshi T, Ohtori S, Inoue G et al (2010) Lumbar posterolateral fusion inhibits sensory nerve ingrowth into punctured lumbar intervertebral discs and upregulation of CGRP immunoreactive DRG neuron innervating punctured discs in rats. Eur Spine $\mathrm{J}$ 19:593-600

16. Lotan R, Al-Rashdi A, Yee A et al (2010) Clinical features of conjoined lumbosacral nerve roots versus lumbar intervertebral disc herniations. Eur Spine J 19:1094-1098

17. Min WK, Bae JS, Park BC et al (2010) Proliferation and osteoblastic differentiation of bone marrow stem cells: comparison of vertebral body and iliac crest. Eur Spine J 19:1753-1760

18. Mulholland RC (2008) The myth of lumbar instability: the importance of abnormal loading as a cause of low-back pain. Eur Spine J 17:619-625

19. Nakamae T, Tanaka N, Nakanishi K et al (2010) Quantitative assessment of myelopathy patients using motor evoked potentials produced by transcranial magnetic stimulation. Eur Spine $\mathrm{J}$ 19:685-690

20. Ohtori S, Koshi T, Yamashita M et al (2010) Existence of pyogenic spondylitis in Modic type 1 change without other signs of infection: 2-year follow-up. Eur Spine J 19:1200-1205

21. Roelofs PD, Van Poppel MN, Bierma-Zeinstra SM et al (2010) Determinants of the intention for using a lumbar support among home care workers with recurrent low back pain. Eur Spine J 19:1502-1507

22. Phillips LA, Caroll LJ, Cassidy JD et al (2010) Whiplash-associated disorders: who gets depressed? Who stays depressed? Eur Spine J 19:945-956

23. Poulain C, Kerneis S, Rozenberg S et al (2010) Long-term return to work after a functional restoration program for chronic lowback pain patients: a prospective study. Eur Spine J 19:1153-1161 
24. Quante M, Lorenz J, Hauck M (2010) Laser-evoked potentials: prognostic relevance of pain pathway defects in patients with acute radiculopathy. Eur Spine J 19:270-278

25. Roelofs PD, Bierma-Zeinstra SM, Van Poppel MNM (2007) Lumbar supports to prevent recurrent low-back pain among home care workers: a randomized trial. Ann Intern Med 147:685-692

26. Scardino FB, Rocha LP, Barcelos AC et al (2010) Is there a benefit to operating on patients (bedridden or in wheelchairs) with advanced stage cervical spondylotic myelopathy? Eur Spine J 19:699-705
27. Silverplats K, Lind B, Zoëga B et al (2010) Clinical factors of importance for outcome after lumbar disc herniation surgery: long-term follow-up. Eur Spine J 19:1459-1467

28. Van Hooff ML, Van der Merwe JD, O'Dowd J et al (2010) Daily functioning and self-management in patients with chronic low back pain after an intensive cognitive behavioral programme for pain management. Eur Spine J 19:1517-1526

29. Zhong WQ, Jiang L, Ma QJ et al (2010) Langerhans cell histiocytosis of the atlas in an adult. Eur Spine J 19:19-22 